Disclosure of Interest: None declared

DOI: 10.1136/annrheumdis-2017-eular.3758

\section{SAT0434 NETWORK META-ANALYSIS ON THE EFFICACY OF NOVEL THERAPEUTIC AGENTS IN PATIENTS WITH PSORIATIC ARTHRITIS}

T.T. Cheung, M.F. Tsoi, Y. Fei, C.S. Lau, B.M.Y. Cheung. Medicine, The University of Hong Kong, Hong Kong, Hong Kong

Background: Novel therapeutic agents are more effective than DMARDs in the management of psoriatic arthritis. However, direct comparisons of efficacy between these novel therapeutic agents are lacking.

Objectives: This network meta-analysis aims to compare the relative efficacies between different novel therapeutic agents.

Methods: Literature searching was conducted in MEDLINE, EMBASE, Scopus, ISI Web of Science, Cochrane Library, Clinicaltrials.gov and recent rheumatology conference abstracts up to Nov 2016. 2 independent researchers analysed the articles. For inclusion, randomised, placebo-controlled trials must report the proportion of patients achieving ACR20, ACR50, ACR70 and PASI75 responses. The outcomes of this network meta-analysis were the proportion of patients achieving ACR20, ACR50, ACR70 and PASI75 responses with reference to placebo and etanercept.

Results were analysed using random effect model by $R$ statistics (version 3.3.1) with statistical package netmeta (version $0.9-2$ ). The heterogeneity of the study results was determined by the $\mathrm{I}^{2}$ statistics.

Results: 18 trials were included into this study. In general, all novel therapeutic agents demonstrated superior efficacies than placebo. With reference to etanercept, apremilast and ustekinumab were associated with less proportions of patients achieving ACR20 response (odds ratio [95\% confidence interval]: 20mg apremilast: 0.18 [0.07-0.48]; 30mg apremilast: 0.24 [0.09-0.62]; 45mg ustekinumab: 0.26 [0.09-0.73]; 90mg ustekinumab: 0.32 [0.11-0.90]).

Etanercept was not different from apremilast, ustekinumab, golimumab, adalimumab, ixekizumab, certolizumab, ixekizumab and secukinumab in terms of ACR20 and ACR50 responses.

Golimumab and infliximab were associated with greater proportions of patients achieving PASI75 response, though the difference did not reach statistical significance. (odds ratio [95\% confidence interval]: golimumab: 3.51 [0.44-28.2]; infliximab: 5.97 [0.89-40.2]).

Table 1. Network meta-analysis on proportion of patients achieved ACR20 and PASI75 response with reference to etanercept

\begin{tabular}{lcc}
\hline & ACR20 response & PASI75 response \\
\hline 10mg Tofacitinib BID & $0.38[0.12 ; 1.22]$ & $0.68[0.13 ; 3.54]$ \\
150mg Secukinumab & $0.64[0.22 ; 1.90]$ & $0.49[0.07 ; 3.19]$ \\
20mg Apremilast BID & $0.18[0.07 ; 0.49]$ & $0.39[0.08 ; 1.83]$ \\
300mg Secukinumab & $0.77[0.21 ; 2.80]$ & $0.67[0.10 ; 4.56]$ \\
30mg Apremilast BID & $0.24[0.09 ; 0.63]$ & $0.48[0.10 ; 2.22]$ \\
45mg Ustekinumab & $0.26[0.09 ; 0.75]$ & $1.18[0.15 ; 9.09]$ \\
5mg Tofacitinib BID & $0.25[0.08 ; 0.81]$ & $0.64[0.12 ; 3.29]$ \\
80mg Ixekizumab Q2w & $0.40[0.12 ; 1.27]$ & $2.74[0.49 ; 15.37]$ \\
80mg Ixekizumab Q4w & $0.35[0.11 ; 1.11]$ & $1.73[0.32 ; 9.31]$ \\
90mg Ustekinumab & $0.32[0.11 ; 0.92]$ & $1.40[0.18 ; 10.74]$ \\
Adalimumab & $0.33[0.13 ; 0.86]$ & $0.77[0.17 ; 3.50]$ \\
Certolizumab & $0.42[0.12 ; 1.39]$ & $\mathrm{NA}$ \\
Etanercept (reference) & 1.00 & 1.00 \\
Golimumab & $0.93[0.25 ; 3.40]$ & $3.51[0.44 ; 28.22]$ \\
Infliximab & $0.74[0.26 ; 2.12]$ & $5.97[0.89 ; 40.22]$ \\
Placebo & $0.10[0.04 ; 0.22]$ & $0.09[0.03 ; 0.35]$ \\
\hline NA: not & &
\end{tabular}

NA: not applicable.

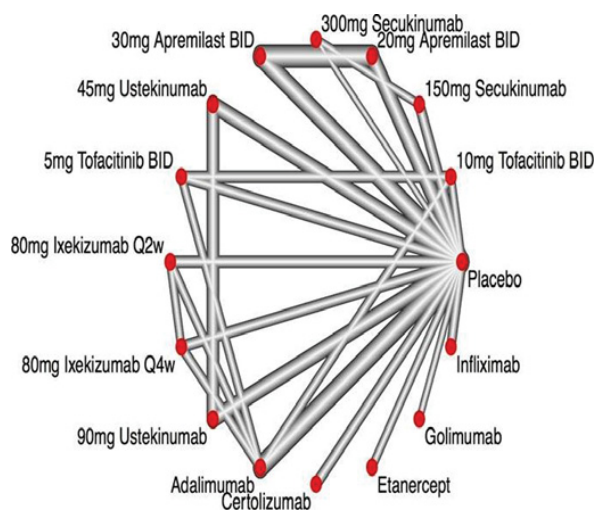

Conclusions: Apremilast and ustekinumab were less efficacious than etanercept in terms of ACR20 response. All the novel therapeutic agents demonstrated comparable efficacies in terms of ACR50, ACR70 and PASI75 responses.

Disclosure of Interest: None declared

DOI: 10.1136/annrheumdis-2017-eular.5096

\section{SAT0435 IL17 CORRELATES POSITIVELY WITH TGF-BETA 1 AND DKK1 AND INVERSELY WITH BMP2 AND 4 IN SYNOVIAL} MEMBRANE OF PATIENTS WITH PSORIATIC ARTHRITIS

J. Pinto-Tasende, M. Fernandez-Moreno, M.E. Vazquez-Mosquera, J.C. Fernandez-Lopez, N. Oreiro-Villar, M. Bejerano-Herrería, B. Acasuso-Pardo de Vera, F.J. Blanco-García. INIBIC-Rheumatology, Complejo Hospitalario Universitario A Coruña, A Coruña, Spain

Background: Immune and non-immune cells contribute to the pathology of chronic arthritis and they can contribute to tissue remodeling and repair as well as disease pathogenesis. An important role for their products such as TGF-beta 1, IL17 or BMPs has been suggested in homeostatic and remodeling mechanisms in arthritis. BMP signaling could have an anti-inflammatory role in the control and maintenance of low levels of pro-inflammatory factors in healthy joints or the early stage of RA.

Objectives: to analyze and compare serum levels, gene expression and immunohistochemistry (IHC) in synovial membrane of inflammation and bone destruction/regeneration biomarkers in patients with psoriatic arthritis (PsA), undetermined seronegative arthritis (USA), Osteoarthritis of the knee (kOA) and ankylosing spondylitis (AS).

Methods: We recruited 45 consecutive patients with chronic knee arthritis referred for undergoing arthroscopies (17 PsA, 12 USA, 12 kOA, 4 AS). Synovial membrane was processed for IHC analysis and quantification of mRNA expression ratio by qRT-PCR. Serum levels of TGF-beta 1, IL6, IL17 and IL22, DKK1, Sclerostin, BMP2, BMP4, Wnt1 and Wnt5a were measured (ELISA). We analyzed and compared these data with the demographic, clinical, analytical and radiological characteristics of the patients. Data were analyzed using the SPSS version 17.0 software and statistical significance was defined as $\mathrm{P}<0.05$.

Results: We obtained valid synovial membrane samples from 41 patients for $\mathrm{IHC}$, RNA extraction and purification from 29 patients for analyze mRNA expression and serum from 38 patients for protein levels measurement. IL17 gene expression was higher in PsA patients $(p=0.027)$ and correlated positively with DKK1 $(r=0.424$, $\mathrm{p}=0.022)$ and negatively with BMP2 $(\mathrm{r}=-0.396, \mathrm{p}=0.033)$ and BMP4 $(\mathrm{r}=-0.472$, $\mathrm{p}=0.010$ ). IHC reactivity for TGF-beta 1 in synovial tissue was higher in patients with psoriatic arthritis ( $p$ 0.010) and correlated positively with IL17 ( $r=0.389$, $p=0.012)$ and DKK1 $(r=0.388, p=0.012)$. Moreover, serum levels of TGF-beta 1 were significantly increased in PsA with erosions $(p=0.044)$.

Conclusions: IL17 gene expression in synovial membrane from patients with psoriatic arthritis was higher than in seronegative undetermined arthritis, osteoarthritis and ankylosing spondylitis patients, correlating positively with DKK1 and negatively with bone morphogenetic proteins 2 and 4 . In addition, TGF-beta in synovial tissue, necessary for the activation of Th17 cells, was higher in patients with psoriatic arthritis, in relation to IL17 and DKK1 increased. Serum TGF beta 1 levels were also higher in patients with erosive disease.

References:

[1] Varas A, Valencia J, Lavocat F, Martínez VG, Thiam NN, Hidalgo L, et al. Blockade of bone morphogenetic protein signaling potentiates the proinflammatory phenotype induced by interleukin-17 and tumor necrosis factor- $\alpha$ combination in rheumatoid synoviocytes. Arthritis Res Ther. 2015;17:192. doi: 10.1186/s13075-015-0710-6.

Acknowledgements: This work has been supported by grant Pl11/00390 from Plan Nacional de Investigación Científica, Desarrollo e Innovación Tecnológica 2008-2011 y cofinanciado por el ISCIII-Subdirección General de Evaluación y Fomento de la Investigación - Fondo Europeo de Desarrollo Regional (FEDER).

Disclosure of Interest: None declared

DOI: 10.1136/annrheumdis-2017-eular.4427

\section{SAT0436 DURABILITY OF APREMILAST RESPONSE IN PATIENTS WITH PSORIATIC ARTHRITIS: LONG-TERM (208-WEEK) RESULTS} FROM THE PALACE 1 TRIAL

A. Kavanaugh ${ }^{1}$, D.D. Gladman ${ }^{2}$, J.J. Gomez-Reino ${ }^{3}$, S. Hall ${ }^{4}$,

E. Lespessailles ${ }^{5}$, P.J. Mease ${ }^{6}$, G. Schett ${ }^{7}$, M. Mcllraith ${ }^{8}$, N. Delev ${ }^{8}$, M. Paris ${ }^{8}$ L. Teng ${ }^{8}$, J. Wollenhaupt ${ }^{9} .{ }^{1}$ UCSD School of Medicine, la Jolla, United States; ${ }^{2}$ Toronto Western Research Institute, Toronto, Canada: ${ }^{3}$ Hospital Clínico Universitario, Santiago, Spain; ${ }^{4}$ Monash University, CabriniHealth, Melbourne, Australia; ${ }^{5}$ University of Orléans, Orléans, France; ${ }^{6}$ Swedish Medical Center and University of Washington School of Medicine, Seattle, United States; ${ }^{7}$ University of Erlangen-Nuremberg, Erlangen, Germany; ${ }^{8}$ Celgene Corporation, Summit, United States; ${ }^{9}$ Schön Klinik Hamburg Eilbek, Hamburg, Germany

Background: Optimizing treatment choice in psoriatic arthritis (PsA) necessitates an understanding of the long-term effects of therapies across varied manifestations of this complex disease. Data from 4 years of apremilast (APR) treatment in PALACE 1 were used to examine disease control across markers of active inflammation, such as SJC, as well as improvements in patient (pt) functionality, as assessed using the HAQ-DI.

Objectives: Evaluate long-term outcomes with APR treatment after $\geq 1$ DMARD or biologic in pts with active PsA.

Methods: Pts were randomized $(1: 1: 1)$ to placebo (PBO), APR $30 \mathrm{mg}$ BID (APR30), or APR $20 \mathrm{mg}$ BID (APR20). The PBO-controlled phase continued to Wk 24, at which time all remaining PBO pts were re-randomized to APR30 or 